
\title{
$\begin{array}{ll}\text { Research Square } & \text { Preprints are preliminary reports that have not undergone peer review. } \\ \text { They should not be considered conclusive, used to inform clinical practice, } \\ \text { or referenced by the media as validated information. }\end{array}$
}

\section{Characteristics and Recovery Methods of Studies Falsely Excluded During Literature Screening -A Systematic Review}

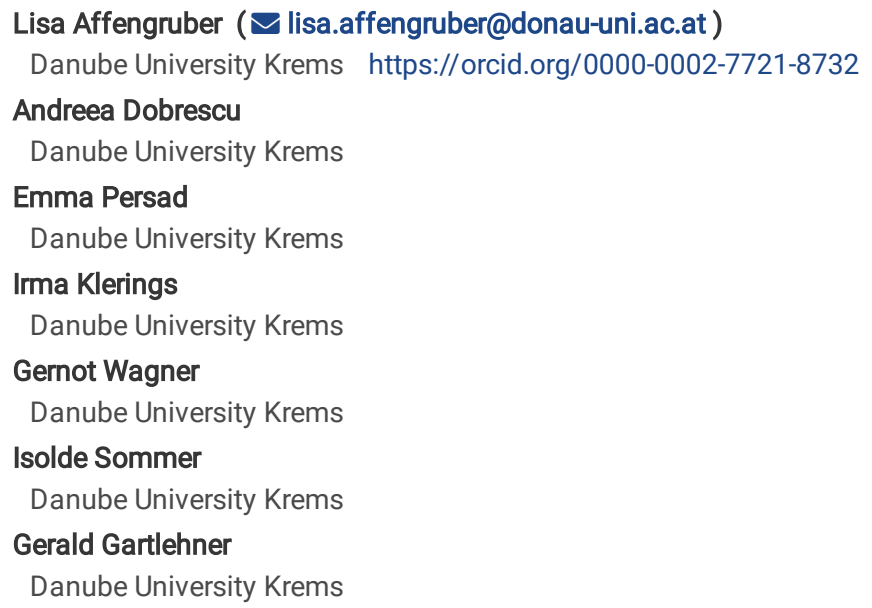




\section{Abstract}

Background: Due to the growing need to provide evidence syntheses under time constraints, researchers have begun focusing on the exploration of rapid review methods, which often employ single-reviewer literature screening. However, single-reviewer screening misses, on average, $13 \%$ of relevant studies, compared to $3 \%$ with dual-reviewer screening. Little guidance exists regarding methods to recover studies falsely excluded during literature screening. Likewise, it is unclear whether specific study characteristics can predict an increased risk of false exclusion. This systematic review aimed to identify supplementary search methods that can be used to recover studies falsely excluded during literature screening. Moreover, it strove to identify study-level predictors that indicate an elevated risk of false exclusions of studies during literature screening.

Methods: We performed literature searches for eligible studies in Medline, Science Citation Index Expanded, Social Sciences Citation Index, Current Contents Connect, Embase, Epistemonikos.org, and Information Science \& Technology Abstracts from 1999 to June 23, 2020. We searched for grey literature, checked reference lists, and conducted hand searches in two relevant journals and similar articles searches current to January 28,2021 . Two investigators independently screened the literature; one investigator performed the data extraction, a second investigator checked for correctness and completeness. Two reviewers assessed the risk of bias of eligible studies. We synthesized the results narratively.

Results: Three methods studies, two with a case study design and one with a case series design, met the inclusion criteria. One study reported that all falsely excluded publications ( $8 \%$ ) could be recovered through reference list checking compared to other supplementary search methods. No included methods study analyzed the impact of recovered studies on conclusions or meta-analyses. Two studies reported that up to $8 \%$ of studies were falsely excluded due to uninformative titles and abstracts, and one study showed that $11 \%$ of non-English studies were falsely excluded.

Conclusions: Due to the limited evidence based on two case studies and one case series, we can draw no firm conclusion about the most reliable and most valid method to recover studies falsely excluded during literature screening or about the characteristics that might predict a higher risk of false exclusion

Systematic review registration: https://osf.io/v2pjr/

\section{Background}

Screening of titles, abstracts, and full-text publications to identify potentially eligible studies is an essential methodological element of any evidence synthesis. To reduce the risk of erroneously excluding relevant studies during literature screening, most international evidence synthesis organizations recommend dual-reviewer screening, that is, having two persons independently screen titles, abstracts, and full texts (1-3).

Dual-reviewer screening, however, is time consuming. Due to the growing need to provide evidence syntheses under time constraints, researchers have begun focusing on the exploration of rapid review methods, which streamline the methodological steps of the systematic review process to provide answers more quickly. Rapid reviews often use single-reviewer screening, whereby each record is screened by only a single investigator. Single-reviewer screening reduces screening time by approximately $60 \%$ when compared to dual-reviewer screening (4). The downside of single-reviewer screening, however, is that it is prone to falsely excluding relevant studies. In a crowd-based randomized controlled trial, Gartlehner et al. reported that single-reviewer abstract screening missed on average $13 \%$ (sensitivity: $86.6 \%$; $95 \%$ confidence interval [Cl], $80.6 \%$ to $91.2 \%$ ) of relevant studies (5). By comparison, dualreviewer screening missed an average of $3 \%$ (sensitivity: $97.5 \% ; 95 \% \mathrm{Cl}, 95.1 \%$ to $98.8 \%$ ) of relevant studies (5). In a recent systematic review by Waffenschmidt et al. on single- versus dual-reviewer screening, the median proportion of relevant but missed studies with single-reviewer screening was $5 \%$ (range 0 to $58 \%$ ) (6).

Consequently, methods to mitigate the risk of erroneous exclusions of relevant studies and to recover falsely excluded studies during the evidence synthesis process are critical to ensure the validity of rapid review results. Table 1 lists several methods that could be used in an attempt to recover falsely excluded studies $(7,8)$. These methods are based on supplementary literature searches that can identify studies with a high likelihood of being relevant to the topic of interest. For example, commonly employed methods include reference list checking of the included studies or of other systematic reviews, or using the "similar articles" function in electronic databases. The principle behind these supplementary searches is that investigators can reconsider the inclusion or exclusion of articles with a high likelihood of being relevant. In some cases, they might identify studies that investigators falsely excluded during single-reviewer literature screening. However, to date, only half of the published rapid reviews conducted reference list checking of the eligible studies (9).

Table 1: Definitions of commonly used methods that could recover falsely excluded studies

\begin{tabular}{|c|c|}
\hline $\begin{array}{l}\text { Reference list checking (backward } \\
\text { citation tracking) }\end{array}$ & Checking the reference lists of the included studies and any relevant systematic revi \\
\hline $\begin{array}{l}\text { Similarity searches (i.e., related } \\
\text { articles) }\end{array}$ & $\begin{array}{l}\text { Using a key article to identify additional relevant articles by using a "similar articles" } \\
\text { databases and search engines (e.g., PubMed, Google Scholar) }(7,8)\end{array}$ \\
\hline $\begin{array}{l}\text { Forward citation tracking of included } \\
\text { studies }\end{array}$ & Using citation indexes for forward citation searching based on a key article (8) \\
\hline Academic search engines & Keyword searches using an academic search engine (e.g., Google Scholar) (8) \\
\hline $\begin{array}{l}\text { Contacting } \\
\text { experts/researchers/companies/ } \\
\text { other stakeholders }\end{array}$ & Contacting individuals and organizations for information about relevant studies (8) \\
\hline
\end{tabular}


Additionally, it would be of interest to researchers to know whether certain types of studies or publications have a higher risk of being falsely excluded than others (10). For example, the publication year could play a role, as abstract reporting standards have changed over time. Older studies might be falsely excluded more frequently during abstract screening since information now considered relevant for an abstract might not have been deemed as important at the time of publication. As another example, one explanation for the false exclusion of studies in the systematic review by Waffenschmidt et al. was that the research question was too vague and largely depended on the interpretation of the reviewer (6).

The aim of this study was to systematically assess which methods have been used to recover studies falsely excluded during literature screening Additionally, we aimed to identify the potential predictors and characteristics of falsely excluded studies.

\section{Methods}

The aim of this systematic review was to address the following key questions (KQs):

KQ 1: How effective are different methods in recovering studies falsely excluded during literature screening?

KQ 2a: What are the characteristics of studies that have been falsely excluded during literature screening?

KQ 2b: Can predictors help identify studies that are at a high risk of being falsely excluded?

This systematic review was conducted according to Cochrane methods (11). We followed the Preferred Reporting Items for Systematic Review and MetaAnalysis (PRISMA) 2020 statement (12). The PRISMA checklist can be seen in Additional file 1. We registered our study protocol „Falsely excluded studies in the literature screening process - a systematic review" at https://osf.io (https://osf.io/5zdpb/). Because we could not identify studies that formally assessed predictors of the false exclusion of records during literature screening, we amended the protocol on June 11, 2021. We expanded the inclusion criteria to also include the study characteristics of falsely excluded studies, even if these characteristics were not formally assessed in a predictive model.

\section{Eligibility criteria}

The a priori-defined eligibility criteria are listed in Table 2 and described in more detail below.

Table 2: Inclusion and exclusion criteria 


\begin{tabular}{|c|c|c|}
\hline & Inclusion & Exclusion \\
\hline \multirow{4}{*}{$\begin{array}{l}\text { KQ 1: Methods to recover falsely } \\
\text { excluded studies }\end{array}$} & - Reference list checking of included studies & \multirow[t]{17}{*}{ Other methods } \\
\hline & - Similarity searches (i.e., related articles) & \\
\hline & - Forward citation tracking of included studies using citation indexes & \\
\hline & - Google Scholar search & \\
\hline \multirow{13}{*}{$\begin{array}{l}\text { KQ 2: Potential predictors of false } \\
\text { exclusion }\end{array}$} & - Contacting experts/researchers/companies/other stakeholders & \\
\hline & - Study design & \\
\hline & - Main objective & \\
\hline & - Sample size & \\
\hline & - Country of conduct & \\
\hline & - Year of publication & \\
\hline & $\begin{array}{l}\text { - Structure and content of abstract (e.g., only title available, no abstract, } \\
\text { uninformative abstract) }\end{array}$ & \\
\hline & - Language of publication & \\
\hline & - Risk of bias & \\
\hline & - Database indexing of studies (e.g., PubMed listing) & \\
\hline & - Publication type & \\
\hline & - Journal in which the study is published & \\
\hline & - Impact factor of journal in which the study is published & \\
\hline \multirow[t]{5}{*}{ Outcomes } & - Proportion of falsely excluded studies that could be recovered (KQ 1) & \multirow[t]{5}{*}{ Other outcomes } \\
\hline & $\begin{array}{l}\text { - Recall, precision, numbers needed to read (NNR) of supplementary } \\
\text { searches (KQ 1) }\end{array}$ & \\
\hline & $\begin{array}{l}\text { - Impact of recovered studies on meta-analysis results and conclusions } \\
\text { (KQ 1) }\end{array}$ & \\
\hline & - Falsely excluded studies by characteristics (KQ 2) & \\
\hline & - Falsely excluded studies by predictors (KQ 2) & \\
\hline \multirow[t]{3}{*}{ Study design } & - Systematic reviews (KQ 1, 2) & \multirow{3}{*}{$\begin{array}{l}\text { Nonempirical publications (e.g., } \\
\text { editorials, letters) }\end{array}$} \\
\hline & - Randomized/nonrandomized trials (KQ 1, 2) & \\
\hline & $\begin{array}{l}\text { - Prospective and retrospective, controlled and uncontrolled } \\
\text { observational studies (KQ 1,2) }\end{array}$ & \\
\hline Date of search & Published 1999 or later & 1998 and earlier \\
\hline Publication language & No restrictions & No restrictions \\
\hline
\end{tabular}

Abbreviations: $\mathrm{KQ}=$ Key question, $\mathrm{NNR}=$ numbers needed to read

We searched for studies assessing the use of supplementary search methods (e.g., forward citation tracking, reference list checking, and web searching) to recover studies falsely excluded during literature screening. These supplementary search methods are defined in Table 1.

Additionally, we searched for studies focusing on the predictors and characteristics of falsely excluded studies, such as those based on study design or publication type. For detailed eligibility criteria, see Table 2.

\section{Information sources}

An experienced information specialist performed searches for eligible studies in Medline (Ovid), Science Citation Index Expanded, Social Sciences Citation Index, Current Contents Connect (all via Web of Science), Embase (Elsevier), Epistemonikos.org, and Information Science \& Technology Abstracts (Ebsco) from 1999 to June 23, 2020. We first developed a search strategy for Ovid Medline and then amended it to fit other electronic databases. We considered 
publications in all languages. According to the peer review of the electronic search strategy (PRESS) statement (12), the electronic Ovid Medline search strategy was peer-reviewed by another information specialist. See Additional file 2 for the database search strategies.

In addition, we searched for grey literature (i.e., unpublished studies) relevant to this systematic review. Potential sources of grey literature included the Open Science Framework (www.osf.io), websites of known organizations that produce rapid reviews (e.g., Canadian Agency for Drugs and Technologies in Health [CADTH]) based on the CADTH Grey Matters Checklist (13) , and dissertation databases (e.g., Digital Access to Research Theses [DART]-Europe). Furthermore, we searched for Cochrane Colloquium abstracts of oral, poster, and workshop presentations and Health Technology Assessment international (HTAi) meeting abstracts.

We manually searched the reference lists of background articles on this topic for any relevant citations that our electronic searches might have missed. Additionally, we hand searched journals that regularly publish methods studies, such as Systematic Reviews and Research Synthesis Methods. If our search retrieved conference abstracts of studies that might have fulfilled our inclusion criteria, we manually searched for further information about these studies (e.g., publications, entries in trial registries, etc.). Additionally, an information specialist conducted similar articles searches for identified key articles in PubMed and Google Scholar and forward citation tracking using Scopus up to January 28, 2021. The search results for the similar article searches are ranked by "similarity" to the key article; the top 20 articles are those categorized as the most similar according to the search algorithm. We exported the top 20 articles and assessed them according to our eligibility criteria. See Additional file 3 for the similar articles searches and forward citation tracking

\section{Study records}

\section{Data management}

Identified citations were stored in an EndNote ${ }^{\circledR}$ X8.2 bibliographic database (Thomson Reuters, New York, NY). All results of the abstract and full-text review, including the exclusion reasons during the full-text review, were recorded in the EndNote database. PDF files of all full-text articles were stored on a server accessible to all members of the review team.

\section{Selection process}

Deduplication of the search results was carried out with EndNote® X8.2 (Thomson Reuters, New York, NY). We developed and pilot-tested abstract and fulltext review forms that reflected our inclusion and exclusion criteria. Two independent reviewers screened abstracts and full-text articles in Covidence (www.covidence.org) and evaluated their eligibility for inclusion. Any discrepancies were resolved through discussion or consultation with a third reviewer. A total of 50 abstracts were piloted by all reviewers to resolve discrepancies and to test the abstract review form. The full-text review was piloted with five full-text articles.

\section{Data collection process}

We designed and pilot-tested a structured data abstraction form. The data were extracted by one reviewer and checked for completeness and accuracy by a second investigator. The data extraction process was piloted with five studies.

\section{Data items}

For studies that met our inclusion criteria, we extracted the following study characteristics and outcomes:

- Study characteristics: author, year of publication, aims, study design, sample size (e.g., number of studies analyzed), number of reviewers involved

- Characteristics of methods/information sources used to recover falsely excluded studies (for KQ 1)

- Characteristics of falsely excluded studies/publications: study design, content of the abstract, language of publication (for KQ 2)

- Outcomes: proportion of falsely excluded studies/publications that could be recovered, impact of recovered studies on meta-analysis results and/or conclusions, proportion of falsely excluded studies/publications by characteristic or predictor

\section{Risk of bias assessment}

For methods studies with a case study design we adapted the Joanna Briggs Institute Critical Appraisal Checklist for Case Reports and for methods studies with a case series design the Joanna Briggs Institute Critical Appraisal Checklist for Case Series (14).

\section{Data synthesis}

We summarized the results narratively and grouped them by outcomes of interest. We did not identify enough studies with a similar design to be able to conduct meta-analyses. 


\section{Results}

The literature searches identified 3750 deduplicated unique records, of which 124 full texts were assessed for eligibility. Three studies published in four publications met our inclusion criteria $(10,15-17)$. One study reported on KQ 1, all three included studies considered KQ 2a, and no study was identified for KQ 2b. Figure 1 depicts the record review flow. Additional file 4 lists the studies excluded at the full-text level and the reasons for exclusion.

\section{Characteristics of the included studies}

We included three studies (published in four articles), of which two were methods studies with a case study design $(10,16,17)$, and one was a methods study with a case series design (15). We rated the risk of bias of two studies as high $(15,17)$ and of one study as low $(16)$. Additional file 5 presents the detailed risk of bias ratings. The aim of the three studies was mainly to assess different methods to accelerate the literature screening process, but they also reported the study characteristics of falsely excluded studies and recovery methods. Rathbone et al. $(15)$ and Feehan et al. $(10,17)$ mentioned uninformative abstract content details as a study characteristic of the falsely excluded studies. The study by Busse et al. (16) reported falsely excluded studies by publication language, comparing English-speaking reviewers with native-speaking reviewers. The study by Feehan et al. additionally reported their methods to recover those studies falsely excluded during literature screening $(10,17)$.

For one methods study with a case study design (17), we identified one companion publication through a conference abstract from Beck et al. (18), the second author of Feehan et al., which was mentioned in a Cochrane review on "Checking reference lists to find additional studies for systematic reviews," and added relevant information (10). Table 3 presents the characteristics of the included studies.

Three methods studies $(10,15-17)$ assessed studies falsely excluded during abstract screening; one study assessed only full-text screening (16). The three studies $(10,15-17)$ assessed one to ten systematic reviews including 53 to 211 studies and involved five to 16 reviewers in their literature screening process. The results for KQs 1 and 2 are described in detail in the following sections and in Table 3.

Table 3: Characteristics and results of the included studies

Abbreviations: $\mathrm{KQ}=$ key question, $\mathrm{N}=$ number, $\mathrm{NA}=$ not applicable, NR= not reported, $\mathrm{PICo}=$ Participants, Intervention, and Comparator, but not the Outcome, $\mathrm{SR}=$ systematic review

\section{Methods to recover studies falsely excluded during literature screening (KQ 1)}

One methods study with a case study design $(10,17)$ conducted a scoping review on an orthopedic topic and analyzed all references included in their review. We rated this study as having a high risk of bias due to it missing conclusions and clear descriptions of the characteristics of falsely excluded studies. The authors found that 11 of 134 eligible studies had been initially found by database searches but had been erroneously excluded during dual screening of the titles and abstracts. The investigators recovered all 11 falsely excluded references (100\%) through reference list checking of the included studies $(10,17)$. The investigators also performed other supplementary search methods, such as contacting key authors, reference list checking, forward citation tracking, and hand searches of online journal websites, but these methods did not recover any falsely excluded references. The study did not analyze the impact of recovered studies on conclusions or meta-analyses.

\section{Characteristics or predictors of falsely excluded studies (KQ 2)}

All three studies explored the study characteristics of studies falsely excluded during literature screening. We could not identify studies that formally assessed whether specific characteristics could predict an increased risk of a study being falsely excluded.

The characteristics mentioned in the studies were uninformative abstract content details and a non-English publication language (Table 3). The following sections summarize these findings in more detail.

\section{Abstract content details}

Two methods studies, one with a case study design and one with a case series design, mentioned uninformative abstract content details as a characteristic for false exclusion $(10,15,17)$. We rated these two methods studies as having a high risk of bias mainly due to them analyzing a convenience case or case series and missing reports of the eligibility criteria and characteristics of the included cases. Feehan et al. (10,17) documented false exclusions during the production of a scoping review including 134 publications. Eleven of the 134 publications (8\%) were falsely excluded due to uninformative titles and abstracts $(10,17)$. Rathbone et al. $(15)$ assessed a convenience sample of 10 systematic review datasets including 211 eligible studies, derived from the literature searches of completed systematic reviews, to test Participants, Intervention, and Comparator, but not the Outcome (PICo)-based title-only screening. Only a single study was falsely excluded by PICo-based title-only single screening in one of the 10 systematic reviews. The authors reported that 'ventilation' was used in the title as an alternative term for oxygen therapy, and this was not listed in the MeSH (Medical Subject Headings) database nor found while searching other resources, and therefore, subject knowledge was needed to identify the study. They concluded that if authors use uncommon or ambiguous terminology in the abstract, this might lead to false exclusion (15).

Page 6/11 


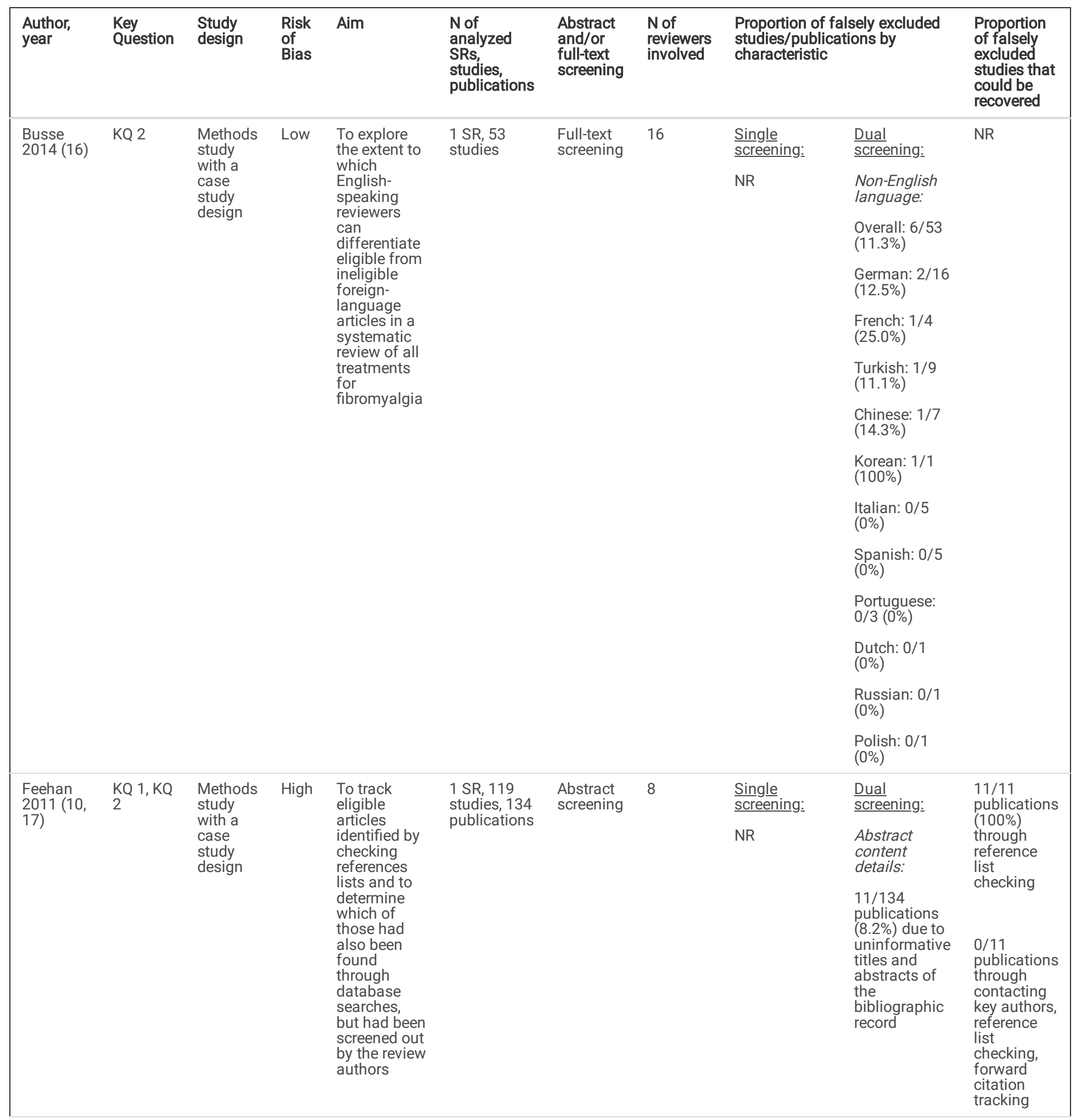




\begin{tabular}{|c|c|c|c|c|c|c|c|c|c|c|}
\hline $\begin{array}{l}\text { Author, } \\
\text { year }\end{array}$ & $\begin{array}{l}\text { Key } \\
\text { Question }\end{array}$ & $\begin{array}{l}\text { Study } \\
\text { design }\end{array}$ & $\begin{array}{l}\text { Risk } \\
\text { of } \\
\text { Bias }\end{array}$ & Aim & $\begin{array}{l}\text { Nof } \\
\text { analyzed } \\
\text { SRs, } \\
\text { studies, } \\
\text { publications }\end{array}$ & $\begin{array}{l}\text { Abstract } \\
\text { and/or } \\
\text { full-text } \\
\text { screening }\end{array}$ & $\begin{array}{l}\mathrm{N} \text { of } \\
\text { reviewers } \\
\text { involved }\end{array}$ & $\begin{array}{l}\text { Proportion of fa } \\
\text { studies/publica } \\
\text { characteristic }\end{array}$ & $\begin{array}{l}\text { sely excluded } \\
\text { ions by }\end{array}$ & $\begin{array}{l}\text { Proportion } \\
\text { of falsely } \\
\text { excluded } \\
\text { studies that } \\
\text { could be } \\
\text { recovered }\end{array}$ \\
\hline $\begin{array}{l}\text { Rathbone } \\
2017 \text { (15) }\end{array}$ & KQ 2 & $\begin{array}{l}\text { Methods } \\
\text { study } \\
\text { with a } \\
\text { case } \\
\text { series } \\
\text { design }\end{array}$ & High & $\begin{array}{l}\text { To evaluate } \\
\text { the } \\
\text { feasibility of } \\
\text { PICo-based } \\
\text { title-only } \\
\text { screening for } \\
\text { scoping } \\
\text { searches } \\
\text { and rapid } \\
\text { reviews by } \\
\text { measuring } \\
\text { the reduction } \\
\text { in screening } \\
\text { effort and } \\
\text { the } \\
\text { maintenance } \\
\text { of recall of } \\
\text { relevant } \\
\text { records }\end{array}$ & $\begin{array}{l}10 \text { SRs, } 211 \\
\text { studies }\end{array}$ & $\begin{array}{l}\text { Abstract } \\
\text { screening }\end{array}$ & 5 & $\begin{array}{l}\text { Single } \\
\text { screening: } \\
\begin{array}{l}\text { Abstract } \\
\text { content } \\
\text { details: }\end{array} \\
1 / 211 \\
(0.005 \%) \text { due } \\
\text { to an } \\
\text { uninformative } \\
\text { title }\end{array}$ & $\begin{array}{l}\frac{\text { Dual }}{\text { screening: }} \\
\text { NR }\end{array}$ & NR \\
\hline
\end{tabular}

\section{Non-English publication language}

One methods study with a case study design, rated as low risk of bias, mentioned a publication language other than English as a characteristic for false exclusion during full-text screening. Busse et al. (16) evaluated a 10-question guide for English reviewers to assess the inclusion of non-English articles compared to native-language speakers. The authors reported false exclusion during the production of one systematic review of randomized controlled trials of fibromyalgia therapy including 53 publications published in 11 languages other than English. Six of the 53 full texts ( $11 \%$ ) were falsely excluded by English-language reviewers due to being published in German (2/53), French, Turkish, Chinese, and Korean (1/53, respectively) languages (16).

\section{Discussion}

To the best of our knowledge, this is the first systematic review that addresses methods to recover falsely excluded studies and study characteristics that can potentially predict a higher risk of false exclusions during literature screening. Our review identified three studies (10, 15-17). One study reported on methods to recover studies falsely excluded during literature screening (KQ 1), all three included studies considered the characteristics of falsely excluded studies (KQ 2a), and no study was identified on predictors that could help identify studies at a high risk of being falsely excluded (KQ 2b). For KQ 1, only one methods study with a case study design that evaluated supplementary search methods was identified. Only reference list checking recovered $100 \%$ of the studies missed by dual literature screening; other supplementary search methods, such as contacting key authors, forward citation tracking, and hand searches of online journal websites, did not recover any falsely excluded studies. The study did not analyze the impact of recovered studies on conclusions or meta-analyses. $(10,17)$. Three studies $(10,15-17)$ reported on the characteristics of falsely excluded studies. Two studies $(10,15,17)$ reported false exclusions of up to $8 \%$ of studies due to uninformative titles and abstracts. Another study (16) reported false exclusion of $11 \%$ of studies due to the nonEnglish publication language.

For rapid reviewers, the results underline the importance of reference list checking as well as considering uninformative titles/abstracts and non-English publications. It seems prudent that rapid review teams should check the reference lists of relevant publications to recover possible missed studies. Other supplementary search methods, such as contacting key authors, forward citation tracking, and hand searches of online journal websites, do not seem to be as effective. However, this evidence is derived from only a single study with a high risk of bias. Review team leaders should consider discussing uninformative titles and abstracts with screeners to avoid such false exclusions, perhaps by requesting that screeners include studies with uninformative titles or abstracts by default for a more in-depth evaluation at the full-text level. Journals and authors should strive to follow abstract reporting guidelines within the theme they are writing, considering the structure and content details of their abstract to ensure their studies are not falsely excluded. Journal editors and peer reviewers should also emphasize informative titles and abstracts. Additionally, the results show that authors should involve translation software or translators in the conduct of rapid and systematic reviews including non-English articles, either internally or through a research network.

Overall, the quality and quantity of the included studies limit the evidence base of our systematic review. We identified three single methods studies on individual cases, assessing only 12 systematic reviews and 18 falsely excluded studies, that mostly address KQ 2 . For KQ 1 , we were only able to identify one methods study with a case study design. For KQ 2, we could only identify studies assessing the characteristics "Abstract content details" and "nonEnglish publication language." We were not able to identify studies addressing other characteristics or predictors of falsely excluded studies. We could not identify studies including predictive models for falsely excluded studies. It might be possible that reviewers falsely excluded studies due to other reasons (e.g., loss of concentration, reviewer's experience) that were not formally addressed by this systematic review. Additionally, the review includes two high risk of bias studies of three included studies. We rated these two studies as having a high risk of bias mainly due to them analyzing a convenience case or case series and missing reports of the eligibility criteria and characteristics of the included cases. These flaws could have an influence on the reliability of our results. Therefore, the results should be cautiously taken into consideration. 
Our systematic review has some methodological limitations. Although we applied a rigorous methodology according to Cochrane methods (11), we cannot rule out the possibility that we might have missed relevant studies. Another known threat to the validity of systematic reviews that we cannot exclude is publication bias. Although we searched for grey literature, relevant research on this topic might not be published due to nonsignificant results.

Based on our findings, there is an immense need for future research to evaluate supplementary search methods to recover studies falsely excluded during literature screening, particularly given the increased demand for rapid reviews employing literature screening shortcuts. No guidance document of any institution has addressed this issue. Studies assessing the overall impact of retrieving missed studies on conclusions and meta-analyses are also warranted. Additionally, further research on the characteristics and predictors of falsely excluded studies is needed. Thus, a methods study that analyzes a heterogeneous data set with a large quantity of screening decisions for possible predictors in a regression analysis or prediction model is warranted.

\section{Conclusions}

We cannot draw any firm conclusion about the most reliable and most valid method to recover studies falsely excluded during literature screening, as the available evidence is limited to a single case study. Furthermore, due to the limited evidence from two case studies and one case series, we can draw no firm conclusions on characteristics that might predict a higher risk of false exclusion.

\section{Abbreviations}

CADTH = Canadian Agency for Drugs and Technologies in Health

$\mathrm{Cl}=$ confidence interval

CONSORT $=$ Consolidated Standards of Reporting Trials

DART $=$ Digital Access to Research Theses

IMRaD = Introduction, Materials and Methods, Results, and Discussion and Conclusion

$\mathrm{KQ}=$ Key question

MeSH = Medical Subject Headings

$\mathrm{N}=$ number

$\mathrm{NA}=$ not applicable

NNR $=$ numbers needed to read

NR $=$ not reported

$\mathrm{OR}=$ odds ratio

PRESS $=$ peer review of the electronic search strategy

PRISMA = Preferred Reporting Items for Systematic reviews and Meta-Analyses

QUOROM = QUality Of Reporting Of Meta-analyses

$\mathrm{RCT}=$ randomized controlled trial

SR = Systematic Review

STARD $=$ Standards for Reporting Diagnostic Accuracy

\section{Declarations}

Ethics approval and consent to participate: Not applicable.

Consent for publication: Not applicable.

Availability of data and materials: All data has been summarized and provided in the manuscript or supplementary files.

Competing interests: The authors declare that they have no competing interests.

Funding: This work was supported by a scholarship for the first author (LA) of the Gesellschaft für Forschungsförderung Niederösterreich m.b.H. 
Authors' contributions: LA, AD, and GG developed the study concept. LA wrote the protocol. IK conducted the literature searches and similar article searches. $L A, A D, G W$, IS, and EP screened the references and extracted the data. LA conducted grey literature searches, reference list checking, and hand searches. GG provided methodological input throughout the study. LA, AD, and EP created the first draft of the manuscript, which GG, GW, IS, and IK critically revised. All authors read and approved the final version of the submitted manuscript.

Acknowledgments: We would like to thank Angela Kaminski-Hartenthaler, who supported the abstract screening. In particular, we want to thank Jos Kleijnen for his input on the manuscript.

\section{References}

1. Aromataris E, Munn Z. JBI Manual for Evidence Synthesis. The Joanna Briggs Institute. 2020;2020.

2. Centre for Reviews Dissemination. CRD's guidance for undertaking reviews in healthcare: York Publ. Services; 2009.

3. Institute for Quality and Efficiency in Health. General Methods Version 6.0 2020. 2020.

4. Shemilt I, Khan N, Park S, Thomas J. Use of cost-effectiveness analysis to compare the efficiency of study identification methods in systematic reviews. Systematic reviews. 2016;5(1):140.

5. Gartlehner G, Affengruber L, Titscher V, Noel-Storr A, Dooley G, Ballarini N, et al. Single-reviewer abstract screening missed 13 percent of relevant studies: a crowd-based, randomized controlled trial. Journal of Clinical Epidemiology. 2020.

6. Waffenschmidt S, Knelangen M, Sieben W, Bühn S, Pieper D. Single screening versus conventional double screening for study selection in systematic reviews: a methodological systematic review. BMC medical research methodology. 2019;19(1):132.

7. European Network for Health Technology Assessment. Process of information retrieval for systematic reviews and health technology assessments on clinical effectiveness. EUnetHTA methodological guidance version 2.0. 2019.

8. Lefebvre C, Glanville J, Briscoe S, Littlewood A, Marshall C, Metzendorf MI, et al. Searching for and selecting studies. Cochrane Handbook for Systematic Reviews of Interventions. 2019:67-107.

9. Tricco AC, Langlois E, Straus SE, Organization WH. Rapid reviews to strengthen health policy and systems: a practical guide: World Health Organization; 2017

10. Horsley T, Dingwall O, Sampson M. Checking reference lists to find additional studies for systematic reviews. Cochrane Database of Systematic Reviews. 2011(8).

11. Higgins JPT TJ, Chandler J, Cumpston M, Li T, Page MJ, Welch VA (editors). Cochrane Handbook for Systematic Reviews of Interventions version 6.2 (updated February 2021). Cochrane, editor2021.

12. Page MJ, McKenzie JE, Bossuyt PM, Boutron I, Hoffmann TC, Mulrow CD, et al. The PRISMA 2020 statement: an updated guideline for reporting systematic reviews. BMJ. 2021;372:n71.

13. Canadian Agency for Drugs and Technologies in Health (CADTH). Grey Matters: a practical tool for searching health-related grey literature. 2019.

14. Moola S MZ, Tufanaru C, Aromataris E, Sears K, Sfetcu R, Currie M, Lisy K, Qureshi R, Mattis P, Mu P. Chapter 7: Systematic reviews of etiology and risk. $\mathrm{JBl} ; 2020$.

15. Rathbone J, Albarqouni L, Bakhit M, Beller E, Byambasuren O, Hoffmann T, et al. Expediting citation screening using PICo-based title-only screening for identifying studies in scoping searches and rapid reviews. Systematic reviews. 2017;6(1):1-7.

16. Busse JW, Bruno P, Malik K, Connell G, Torrance D, Ngo T, et al. An efficient strategy allowed English-speaking reviewers to identify foreign-language articles eligible for a systematic review. Journal of clinical epidemiology. 2014;67(5):547-53.

17. Feehan LM, Beck CA, Harris SR, Maclntyre DL, Li LC. Exercise prescription after fragility fracture in older adults: a scoping review. Osteoporosis international. 2011;22(5):1289-322.

18. Beck C. The scoping study in physical therapy: application of traditional systematic review guidelines to an emerging methodology. Canadian Health Libraries Association Conference in Kingston; Ontario2010.

\section{Figures}




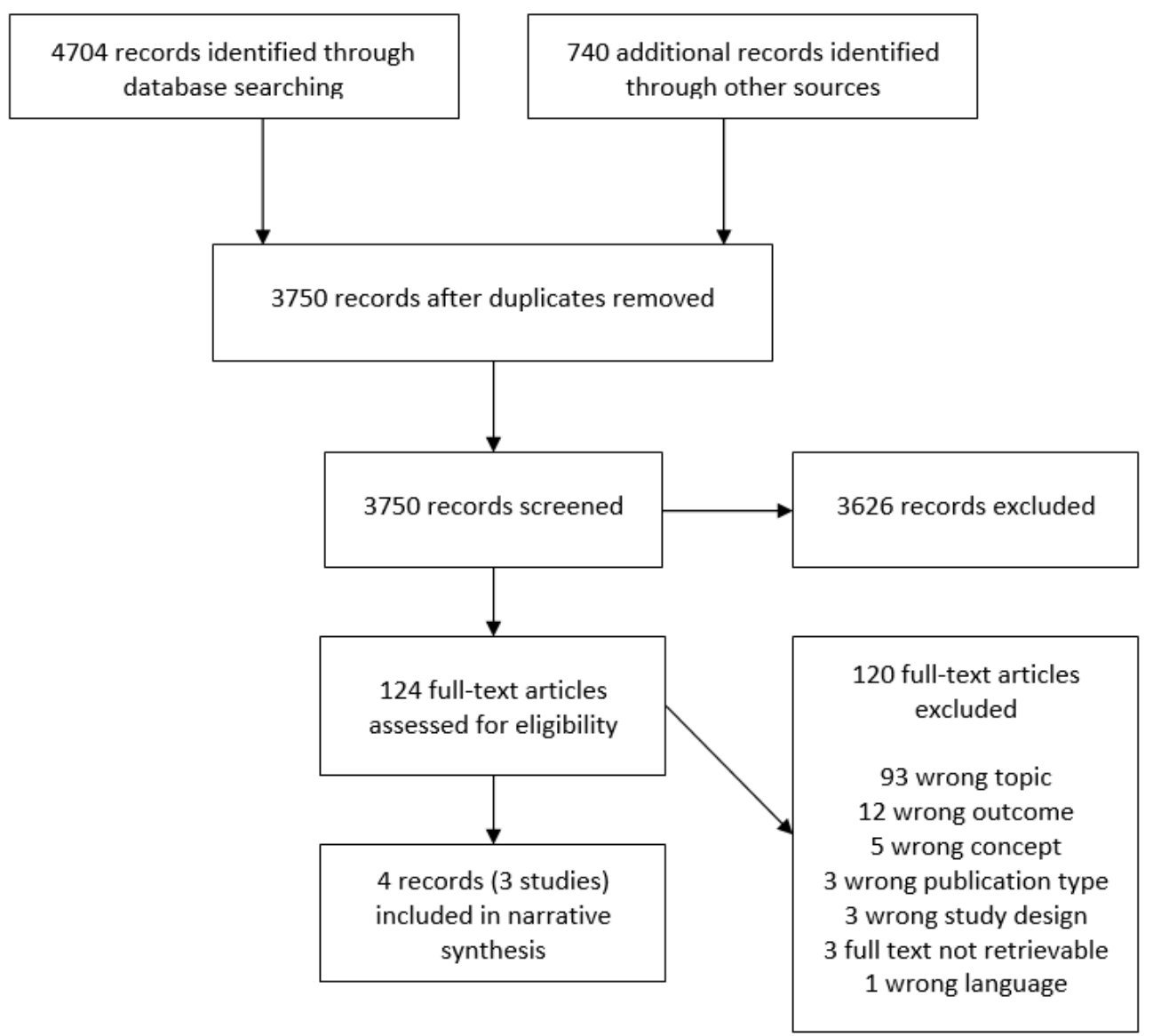

Figure 1

Flow diagram

\section{Supplementary Files}

This is a list of supplementary files associated with this preprint. Click to download.

- Additionalfile1.docx

- Additionalfile2.docx

- Additionalfile3.docx

- Additionalfile4.docx

- Additionalfile5.docx 